\title{
ARTICLE
}

Received 14 Aug 2014 | Accepted 9 Sep 2014 | Published 13 Oct $2014 \quad$ DOI: 10.1038/ncomms6192

\section{Dual-comb spectroscopy based on quantum-cascade-laser frequency combs}

Gustavo Villares', Andreas Hugi', Stéphane Blaser ${ }^{2}$ \& Jérôme Faist ${ }^{1}$

Dual-comb spectroscopy performed in the mid-infrared-where molecules have their strongest rotovibrational absorption lines-offers the promise of high spectral resolution broadband spectroscopy with very short acquisition times ( $\mu s)$ and no moving parts. Recently, we demonstrated frequency comb operation of a quantum-cascade-laser. We now use that device in a compact, dual-comb spectrometer. The noise properties of the heterodyne beat are close to the shot noise limit. Broadband $\left(15 \mathrm{~cm}^{-1}\right)$ high-resolution $(80 \mathrm{MHz}$ ) absorption spectroscopy of both a GaAs etalon and water vapour is demonstrated, showing the potential of quantum-cascade-laser frequency combs as the basis for a compact, all solid-state, broadband chemical sensor.

\footnotetext{
${ }^{1}$ Institute for Quantum Electronics, ETH Zurich, Zurich CH-8093, Switzerland. ${ }^{2}$ Alpes Lasers SA, 1-3 Max.-de-Meuron, C.P. 1766, Neuchâtel CH-2000, Switzerland. Correspondence and requests for materials should be addressed to G.V. (email: gustavo.villares@phys.ethz.ch) or to J.F. (email: jfaist@phys.ethz.ch).
} 
O ptical frequency combs were first introduced in the context of frequency metrology to bridge the gap between radio frequencies and optical frequencies ${ }^{1}$. Since then, several spectroscopy techniques using frequency combs have been demonstrated in various parts of the optical spectrum ${ }^{2}$. In the context of molecular spectroscopy, dual-comb spectroscopy ${ }^{3-9}$ was proposed as an intriguing form of Fourier transform spectroscopy technique, showing a dramatic reduction in measurement times. The mid-infrared part of the optical spectrum is of paramount importance for molecular spectroscopy as the fundamental rotovibrational bands of most light molecules lie in this spectral range, with absorption strength orders of magnitude higher than in the visible or near-infrared ${ }^{10}$. Different approaches have been pursued to obtain mid-infrared frequency combs ${ }^{11}$, directly using transition metals incorporated into chalcogenide hosts ${ }^{12,13}$ or Thulium-doped silica fibre lasers ${ }^{14}$, or indirectly using nonlinear conversion of nearinfrared frequency combs ${ }^{15-20}$. However, these techniques tend to lead to relatively large set-up footprints, with the exception perhaps of microresonator-based combs that recently achieved operation in the mid-infrared ${ }^{21-23}$. Another possibility is a dualcomb-based technique combined with Raman spectroscopy that has recently been demonstrated ${ }^{24,25}$. Consequently, in order to widen the applications of frequency combs to the mid-infrared region of the spectrum, a semiconductor-based mid-infrared dual-comb spectrometer is of high interest for its simplicity and compactness.

Quantum-cascade-lasers (QCLs) ${ }^{26}$ have become the standard semiconductor laser source available in the mid-infrared range, achieving continuous-wave operation at room temperature ${ }^{27}$ with watts of output power ${ }^{28}$. As QCLs are based on intersub-band transitions exhibiting an atomic-like joint density of states, heterogenous stacks ${ }^{29}$ of active regions can be combined, achieving very large spectral coverage ${ }^{30}$ when based on individual broadband active regions designs ${ }^{31}$.

Fundamental mode-locking of QCLs has led to intense

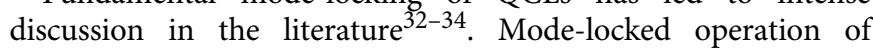
QCLs is difficult to achieve but have been demonstrated at cryogenic temperatures in the mid-infrared in a design where the upper-state lifetime was significantly increased using a highly diagonal laser transition ${ }^{32}$ and in the terahertz where that lifetime is naturally much longer ${ }^{35}$.

Recently, we have demonstrated room temperature, frequency comb operation of a QCL $^{36}$ with frequency-modulated (FM) output characteristics. In this work, we present a compact, semiconductor-based, electrically driven dual-comb spectrometer based on QCL frequency combs. As a proof of principle, we demonstrate broadband high-resolution absorption spectroscopy of a GaAs etalon showing broad absorption features as well as of water vapour at atmospheric pressure. Furthermore, we use the dual-comb set-up to demonstrate the equidistance of the comb teeth down to the $\mathrm{mHz}$ level.

\section{Results}

Mid-infrared QCL frequency combs. Optical frequency combs are characterized by their perfectly equally spaced modes in the frequency domain, in which each individual comb line can be expressed as $f_{n}=f_{\text {ceo }}+n f_{\text {rep }}$, where $n$ is an integer, $f_{\text {rep }}$ is the comb line spacing (also called intermode beat) and $f_{\text {ceo }}$ is the carier-envelope offset frequency.

As discussed in our previous work ${ }^{36}$, the phase-locking mechanism of a free-running, continuous-wave broadband QCL was identified to be four-wave mixing ${ }^{37}$, which, combined with the short gain recovery time of a QCL, leads to a phase signature comparable to a FM laser. This interpretation has been recently confirmed by simulations of the mode dynamics using MaxwellBloch equations ${ }^{38}$. Nevertheless, a flat broadband gain as well as a very low group velocity dispersion are required to achieve phaselocking.

In this work, we employ two QCL frequency combs based on an InGaAs/InAlAs broadband design. They achieve continuouswave operation at room temperature with several $\mathrm{mWs}$ of output power. The comb line spacing of a QCL comb is determined by the length of the device and by its group refractive index. Therefore, the comb line spacing can be easily fixed between few $\mathrm{GHz}$ (long devices) up to several tens of $\mathrm{GHz}$ (short devices). We used 6-mm-long devices, corresponding to comb line spacings of $\sim 7.5 \mathrm{GHz}\left(0.25 \mathrm{~cm}^{-1}\right)$.

The optical spectra of the combs are measured with a Fourier transform infrared spectrometer $\left(0.12 \mathrm{~cm}^{-1}\right.$ resolution) and are represented in Fig. 1a. The combs span $50 \mathrm{~cm}^{-1}$ with a comb line spacing of $0.25 \mathrm{~cm}^{-1}$, resulting in 200 lines for each comb. In contrast to conventional frequency combs based on mode-locked lasers, the optical spectrum of FM combs does not show a gaussian envelope ${ }^{7}$, rather a complex amplitude distribution.

A very unique feature of QCL combs is the fact that the comb line spacing can be measured as an RF modulation arising directly on the laser-biasing current, and extracted from the device through a bias-tee. In fact, the electronic population dynamic in QCLs has an extremely high bandwidth because of the short intersub-band lifetimes ( $0.6 \mathrm{ps})$. Any RF modulation of the optical field is therefore reflected on the electronic population and thus on the current. We therefore found a clear correspondence between the comb line spacing measured electrically and with an optical detector ${ }^{35,36}$. The intermode beat notes of the two frequency combs measured with a spectrum analyser are shown in Fig. 1b. We observe a first intermode beat at $7.451 \mathrm{GHz}$ and the second at $7.464 \mathrm{GHz}$, spaced by $12.6 \mathrm{MHz}$. Both intermode beats show extremely narrow linewidths $(<500 \mathrm{~Hz}$, resolution bandwidth (RBW) limited), which is a signature of the phaselocking mechanism of the combs.

As a reference, we also drove the lasers in a high phase-noise regime by increasing the laser current ${ }^{36}$. This is the configuration encountered in conventional Fabry-Pérot devices ${ }^{33,39}$. The intermode beat is displayed in Fig. 1c and shows a high phasenoise pedestal (few $\mathrm{MHz}$ wide), with a lower phase-noise peak. The spacing between the two intermode beat note frequencies was increased in this regime in order to better separate them.

Multi-heterodyne beat and dual-comb spectroscopy. Figure $2 \mathrm{a}$ shows a schematic of the principle of dual-comb spectroscopy. The technique is based on a multi-heterodyne beating of two frequency combs with slightly different comb line spacings $\left(f_{\text {rep }, 1}\right.$ and $f_{\text {rep, } 2}=f_{\text {rep }, 1}+\Delta f$, respectively, where $\Delta f$ is the difference in the comb line spacings). A detector is then used to measure the multi-heterodyne beat. As shown schematically in Fig. 2a, each line of the first comb will beat with all lines of the second comb creating several different beats in the RF domain. $\Delta f$ must be chosen such as to obtain a one-to-one mapping between the lines of the two combs and their RF counterpart (see Supplementary Fig. 1 and Supplementary Note 1).

In most configurations of dual-comb spectroscopy ${ }^{7}$, one comb is used as a local oscillator, while the other is used to interrogate a sample, as shown in Fig. 2b. Owing to the one-to-one mapping, each multi-heterodyne beat contains information regarding the sample absorption at the optical frequency of the comb line interrogating the sample. As the technique relies on the discrete nature of a frequency comb, the sample absorption is measured at frequencies corresponding to the comb spacing $\left(7.5 \mathrm{GHz}=0.25 \mathrm{~cm}^{-1}\right)$, defining thus the resolution. For the 

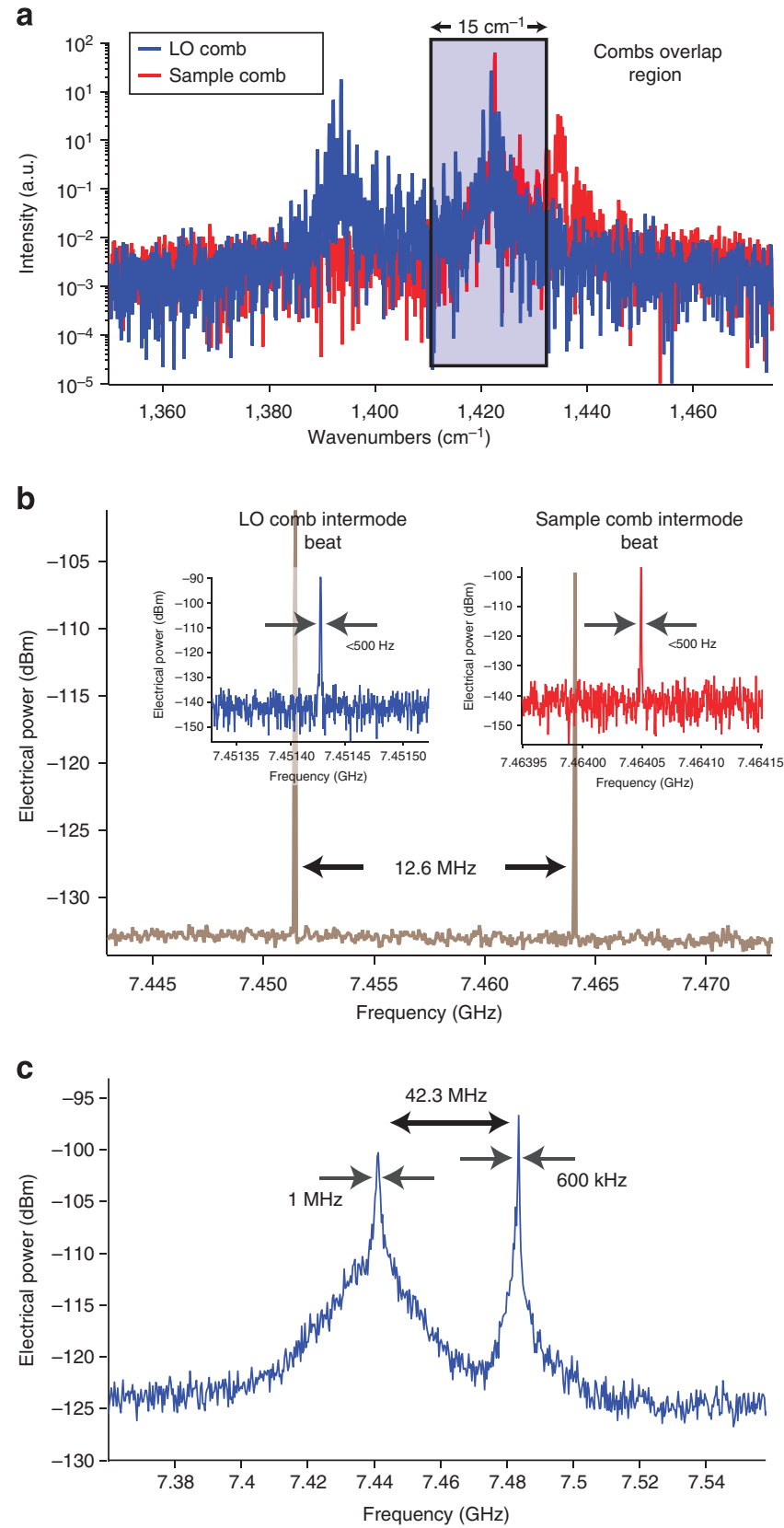

Figure 1 | QCL frequency combs beat notes. (a) Optical spectrum of the local oscillator (LO) comb (blue) and the sample comb (red) measured with a Fourier transform infrared spectrometer (FTIR; $0.12 \mathrm{~cm}^{-1}$ resolution). $I_{\mathrm{LO}}=544 \mathrm{~mA}, T=-13.25^{\circ} \mathrm{C}$ and $I_{\text {signal }}=568 \mathrm{~mA}, T=4{ }^{\circ} \mathrm{C}$. The combs overlap region of the spectrum spans over $15 \mathrm{~cm}^{-1}$. (b) Electrical intermode beat of the $\mathrm{LO}$ and signal combs in the so-called comb regime, measured with a spectrum analyser ( $\mathrm{span}=30 \mathrm{MHz}, \mathrm{RBW}=3 \mathrm{kHz}$ ). Insets: same measurement with a higher resolution ( $\mathrm{span}=200 \mathrm{kHz}$,

$\mathrm{RBW}=500 \mathrm{~Hz}$ ). (c) Electrical intermode beat of $\mathrm{LO}$ and signal combs in a high phase-noise regime, measured with a spectrum analyser $($ span $=200 \mathrm{MHz}, \mathrm{RBW}=20 \mathrm{kHz}$.

spectroscopy of large organic molecules or liquids, this resolution is usually sufficient. However, for gas spectroscopy of small molecules at low pressures, the molecule absorption lines are usually narrower (hundreds of $\mathrm{MHz}$ ) than our comb spacing. As a special feature of QCL combs, the comb teeth can furthermore be swept over one comb spacing by tuning either its temperature or current.
A dual-comb spectroscopy set-up is realized with the two QCL frequency combs previously described and is depicted in Fig. 2b (see Methods for further details). The difference in comb spacings $\Delta f$ can be set between 5 and $40 \mathrm{MHz}$ by changing the temperature and current of both combs. The overlap region between the two combs spans around $15 \mathrm{~cm}^{-1}$ and is represented in Fig. 1a. We implemented a dual detection technique as it helps to remove technical noise on the detected amplitude ${ }^{40}$.

No optical filter was used to spectrally select the overlap region. Note that the overlap region could be increased by using bettermatched devices (using, for example, adjacent chips on the wafer, which was not the case for this work). The output power of the lasers is $\sim 5 \mathrm{~mW}$ and the power on the detector is in general tens of $\mu \mathrm{W}$ as the beams are attenuated before the detection, to minimize detector nonlinearities.

A typical time domain interferogram generated by a multiheterodyne beat of the two QCL combs is shown in Fig. 2c,d. As opposed to the interferogram of conventional dual-comb set-ups where a burst at zero delay time is observed ${ }^{7}$, the beating of our two FM combs shows an almost constant amplitude.

Figure 3a shows a single multi-heterodyne spectrum (acquisition time $\tau_{\mathrm{acq}}=3.2 \mu \mathrm{s}$, in red) as well as an averaged multiheterodyne spectrum (total acquisition time $=100 \mathrm{~ms}$, averaged over 30,517 spectra, in blue), where $\Delta f=9.58 \mathrm{MHz}$. A detailed description of data analysis used for computing the multiheterodyne spectrum is given in the Supplementary Methods. The multi-heterodyne spectrum of the two combs spans $900 \mathrm{MHz}$ with around $N \sim 100$ lines spaced by $\Delta f=9.58 \mathrm{MHz}$, corresponding to an optical bandwidth of $\sim 25 \mathrm{~cm}^{-1}$. Several beat notes can already be observed on the multi-heterodyne spectrum acquired over $\tau_{\text {acq }}=3.2 \mu \mathrm{s}$.

In order to achieve high signal-to-noise (SNR) ratio, the single acquisition time $\tau_{\text {acq }}=3.2 \mu$ s should be shorter than the relative coherence time $\tau_{\text {rel }}$ between the two combs, equal to inverse of the linewidth of an individual multi-heterodyne beat note. As shown in Fig. 3c, the latter is $100 \mathrm{kHz}$ with a sweep time of $30 \mathrm{~ms}$, corresponding to $\tau_{\text {rel }}=10 \mu \mathrm{s}$. As this relative coherence time is expected to grow significantly with shorter acquisition time, our condition $\tau_{\text {acq }}<<\tau_{\text {rel }}$ for coherent accumulation of the signal is well satisfied. Note also that the acquisition time $\tau_{\text {acq }}=3.2 \mu \mathrm{s}$ satisfies the condition $\tau_{\text {acq }}>2 / \Delta f=158$ ns needed to resolve the individual multi-heterodyne beat notes. We could therefore average all the multi-heterodyne spectra after aligning them numerically (see Supplementary Fig. 2 and Supplementary Methods).

As shown in Fig. 3a, all the individual beat notes are visible and above the noise floor on the averaged multi-heterodyne spectrum, demonstrating the high sensitivity of such a multi-heterodyne technique, with more than $50 \mathrm{~dB}$ in difference in power between the different beat notes. The heterodyne efficiency is difficult to be evaluated because of the important line-by-line amplitude variation. It is likely that the SNR ratio of this measurement can still be increased by improving the beam quality on the detector.

To investigate the applicability of the results of ref. 41 to broadband spectroscopy, we measure the multi-heterodyne beat when both QCLs are driven in a high phase-noise regime. Figure $3 \mathrm{~b}$ shows the multi-heterodyne spectrum in this configuration (here $\Delta f=42.3 \mathrm{MHz}$, as in Fig. 1c). As a result of the additional phase-noise, the different multi-heterodyne beat notes display a large overlap, introducing an important cross talk between them. Increasing the frequency difference between the two comb spacings would address this issue at the cost of a much reduced optical frequency coverage. While narrowband spectroscopy has been successfully demonstrated with Fabry-Pérot devices operated in a high phase-noise regime ${ }^{41}$, 
a

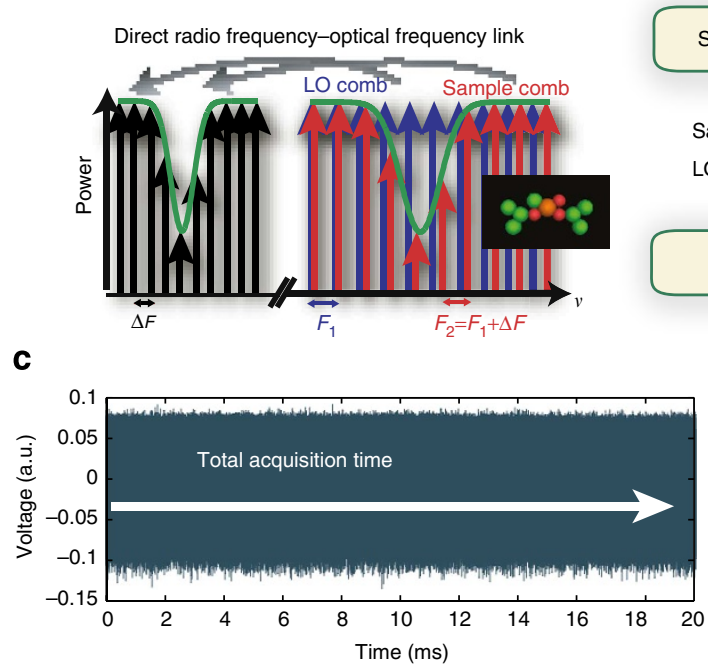

b

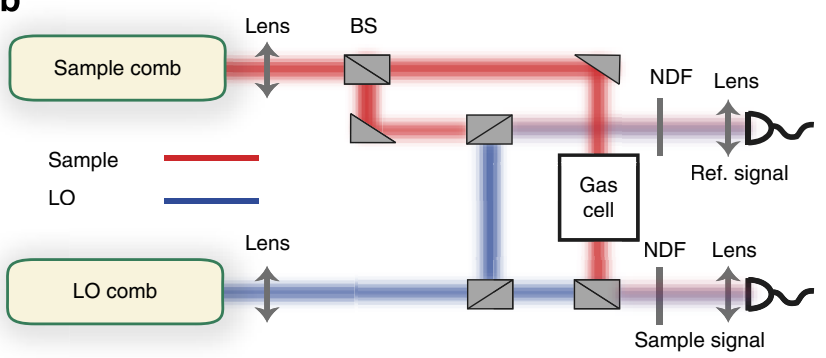

d

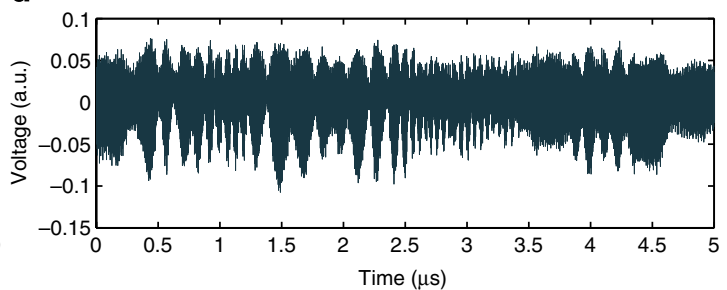

Figure 2 | Principle of dual-comb spectroscopy. (a) A multi-heterodyne beat of two frequency combs with slightly different comb spacings is created on a fast detector, generating a direct link between the optical and the RF domain. The multi-heterodyne beat signal contains information on the sample absorption. (b) Schematic view of the dual-comb spectroscopy set-up based on QCL frequency combs. One comb is used as a LO, while the other interrogates the gas cell. BS: 50-50 Antireflection-coated beam splitter, NDF: neutral density filter. (c) Typical time domain interferogram generated by a multi-heterodyne beat created by the beating of two QCL frequency combs. The beating of our two FM combs shows an almost constant amplitude.

(d) Expanded version (4,000 times) of the same time domain interferogram.

our measurement demonstrates the advantage of QCL frequency combs for dual-comb spectroscopy rather than conventional Fabry-Pérot devices.

Noise properties. The multi-heterodyne set-up serves not only as a spectroscopic tool but also allows for the quantification of important comb characteristics using metrologically accepted counting techniques ${ }^{21}$. Since this set-up effectively maps down the frequency comb from optical domain to the RF frequencies, it allows to measure the equidistance of individual comb lines using frequency counters. The uniformity of the mode spacing can be quantified by evaluating the deviation from equidistant mode spacing $\epsilon$, defined as $^{21}$ :

$$
\epsilon=\frac{f_{M}-f_{0}}{M}-\frac{f_{N}-f_{0}}{N} .
$$

Where $f_{0}, f_{N}$ and $f_{M}$ are the frequencies of three multiheterodyne beat notes. In our measurement, we have set $\Delta f=8$ $\mathrm{MHz}$, giving $f_{0}=30 \mathrm{MHz}, N=5, f_{N}=70 \mathrm{MHz}, M=18$ and $f_{M}=158 \mathrm{MHz}$.

The set-up is illustrated in Fig. 4a (see Methods for further details). After detecting the multi-heterodyne beat signal, three electrical band-pass filters are used to isolate the beat notes at frequencies $f_{0}, f_{N}$ and $f_{M}$. After amplification, three frequency counters are used to measure the frequency oscillations of the beat notes.

In this experiment, we implement an active stabilization (by acting on the temperature of one device) to correct the slow frequency drifts of the multi-heterodyne beat spectrum, in effect to prevent them from drifting out of the bandwidth of the electrical filters (see Methods). Figure $4 \mathrm{~b}$ shows the frequency oscillations of $f_{N}$ (centred at $70 \mathrm{MHz}$ ). The amplitude of the frequency drifts are less than $30 \mathrm{kHz}$ over $30 \mathrm{~s}$, which is significantly lower than the bandwidth of the filters. A precise control of both trigger and gate time of all the counters was implemented (see Methods). It is particularly important as any drift of $f_{\text {ceo }}$ will be seen as a nonuniformity of the comb line spacing if the counters are not perfectly synchronized. Even though the comb is not fully stabilized and $f_{\text {rep }}$ drifts, $\epsilon$ should be zero at any point of time for a perfectly synchronized set-up and a perfectly spaced comb.

Figure $4 \mathrm{c}$ shows the distribution of $\epsilon$ over the entire measurement (gate time $=10 \mathrm{~ms}, 2,523$ counts). We measure a mean value of $\bar{\epsilon}=(-5.6 \pm 32) \mathrm{mHz}$. Normalized to the optical carrier frequency $(42.8 \mathrm{THz}$ or $7 \mu \mathrm{m})$, this gives an accuracy of the equidistance of $7.5 \times 10^{-16}$. The Allan deviation of $\epsilon$ is shown in Fig. $4 \mathrm{~d}$. These measurements definitely prove the equidistance of the mode spacing of a QCL frequency comb.

Amplitude noise of each hererodyne beat note limits the sensitivity of a dual-comb absorption measurement. The SNR achieved with dual-comb spectroscopy has been investigated systematically in the context of conventional dual-comb spectroscopy by Newbury et al. ${ }^{40}$ While the latter analysis is also valid for our set-up, note, however, that dual-comb spectroscopy with FM combs has a fundamental advantage in terms of SNR since, for a given average power, we reduce the peak power incident on the detector, improving therefore the available dynamical range.

The amplitude noise of each of the multi-heterodyne beat notes from the spectra shown in Fig. 5a are investigated. Figure 5b shows the normalized amplitude oscillations of the beat note having the highest amplitude as a function of time, corresponding to the 11 (green-marked) peak highlighted on Fig. 5a. We observe slow fluctuation on top of high frequency noise, with an amplitude of roughly $3 \%$. The Allan deviation of the normalized amplitude oscillations of the highest and one of the lowest amplitude beat notes are shown in Fig. $5 c$ as a function of the integration time ( $40 \mathrm{~ms}$ of total acquisition time). In addition, we represent the Allan deviation induced by the noise floor as well as by the photon noise (see Methods, Supplementary Fig. 3 and Supplementary Note 2). These measurements reveal that the magnitude of the amplitude noise of the multi-heterodyne beats is close to the shot noise limit, as shown on both plots on Fig. $5 \mathrm{c}$. 

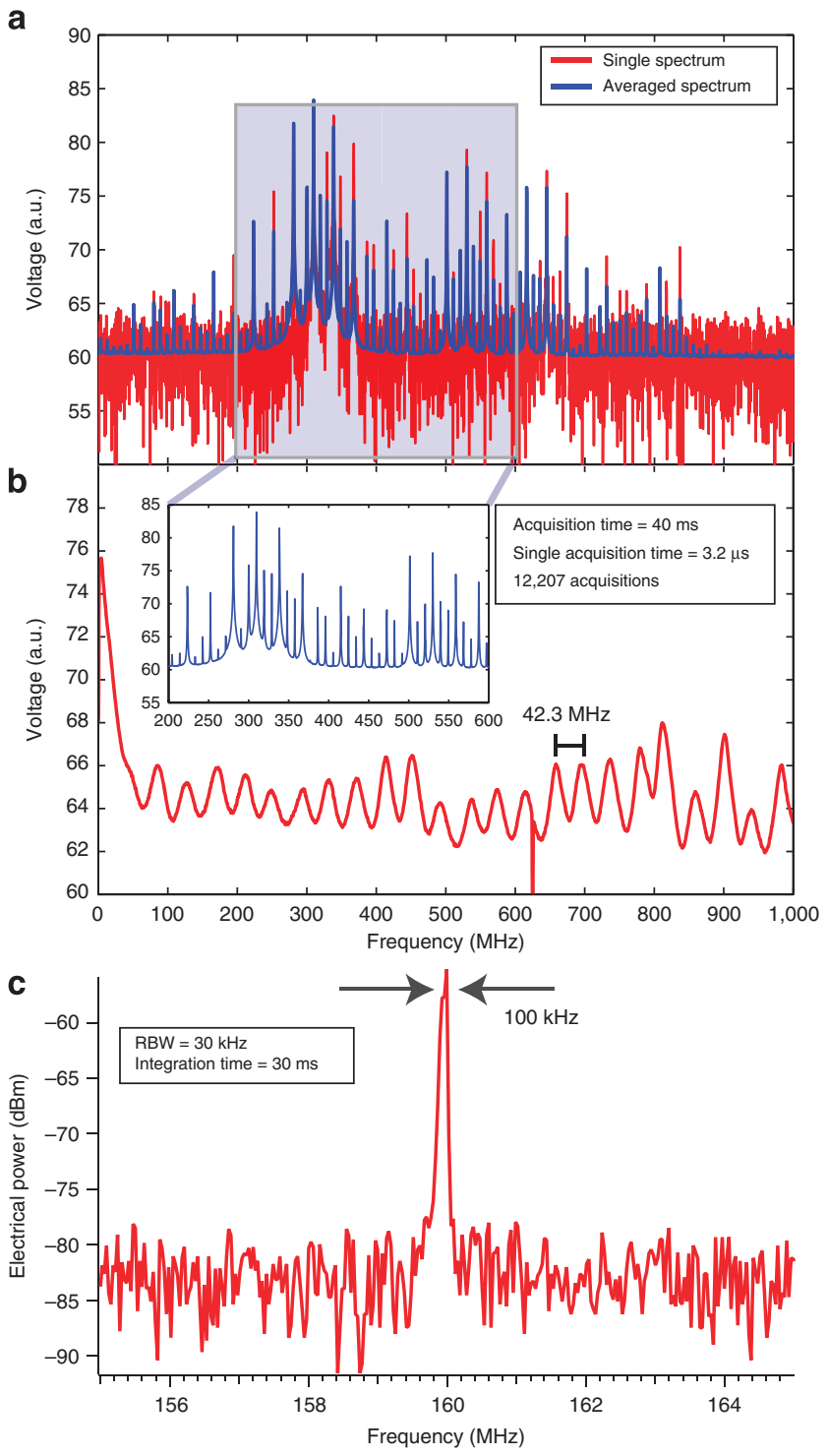

Figure 3 | Multi-heterodyne beat spectra. (a) Single multi-heterodyne spectrum ( $\tau_{\text {acq }}=3.2 \mu \mathrm{s}$; red) and averaged (blue) multi-heterodyne spectrum (total acquisition time $=100 \mathrm{~ms}$, averaged over 30,517 samples) acquired with two QCLs in the comb regime (intermode beat spectrum shown in Fig. 1b). (b) Averaged multi-heterodyne spectrum (total acquisition time $=40 \mathrm{~ms}$, averaged over 12,207 samples) where the two lasers are driven in an increased phase-noise regime (see Fig. 1c). Inset: zoom of the averaged multi-heterodyne spectra of two QCLs driven in the comb regime. (c) Single line of the multi-heterdoyne beat spectrum displayed in Fig. 3a, measured with a spectrum analyser (sweep time $=30 \mathrm{~ms}$, RBW $=30 \mathrm{kHz}$.

In both cases, we observe that the Allan deviations decrease with the integration time. However, the Allan deviation of the high amplitude peak increases at $\sim 200 \mu \mathrm{s}$, corresponding to a technical noise that can be related to the low frequency oscillation observed in Fig. 5b. As the sensitivity of the low-amplitude peak is no longer sufficient to observe this technical noise, the corresponding Allan deviation decreases monotonically with the integration time. Although these measurements do not allow a direct evaluation of the importance of mode partition noise ${ }^{42}$, the fact that our experimental data lie close to simulations where only shot noise is taken into account indicates that mode partition noise adds, in the worst case, only $5-10 \mathrm{~dB}$ to the SNR of the system. These measurements prove that the QCL-based dual-comb set-up is an efficient spectroscopic tool with a SNR close to shot noise limit.

Broadband high-resolution spectroscopy. As a first test of broadband spectroscopy, we first measure the transmission $T$ of a low-finesse etalon consisting of a $450 \mu \mathrm{m}$-thick GaAs double-sided polished wafer. With a refractive index of $\sim 3.3$ at $7 \mu \mathrm{m}$ wavelength, a $450 \mu \mathrm{m}$-thick GaAs etalon shows a periodic transmission with a period of $3.3 \mathrm{~cm}^{-1}$. As discussed before, the etalon transmission acquired by our dual-comb set-up will be sampled at the comb spacing $\sim 0.25 \mathrm{~cm}^{-1}$. We therefore improved the resolution of our set-up by performing a frequency sweep of the combs and interleaving several spectra (see Supplementary Fig. 4 and Supplementary Methods). Note that because we measure the frequency tuning of the LO and sample combs directly in the electrical domain, the frequency accuracy of the measurement is equal to the long-term stability of a single laser, that is, below $10 \mathrm{MHz}$. For practical reasons concerning this frequency sweep, the QCL combs had to be operated in a temperature region where the overlap spectra was $15 \mathrm{~cm}^{-1}$ rather than $25 \mathrm{~cm}^{-1}$.

Figure 6a shows the transmission of the etalon measured with our dual-comb spectrometer $\left(0.25 \mathrm{~cm}^{-1}\right.$ comb spacing swept with a $80-\mathrm{MHz}$ step), together with a fit performed using the expression of the transmission of an etalon. The fitting algorithm uses the etalon length $L$ as fitting parameter and assumes fixed values for the refractive index of the material. We observe the periodic transmission of the etalon over a bandwidth of $15 \mathrm{~cm}^{-1}$. The value of the etalon length computed by the fitting algorithm is $446 \mu \mathrm{m}$, in agreement with the measured thickness. The QCL combs are not actively stabilized while the transmission measurement is performed and slow drifts are still detrimental for the quality of the interleaved transmission.

As a proof of principle applied to gas sensing, we performed transmission measurement of water vapour in air at atmospheric pressure in a $6-\mathrm{cm}$-long gas cell. We applied the same frequency sweep to the combs as described below $(80 \mathrm{MHz}$ step over a bandwidth equal to one comb line spacing). In order to avoid parasitic fringes coming from residual reflectivities in the beam path, we measured the multi-heterodyne beat signal with the gas cell filled with water vapour and subsequently with nitrogen, at each step of the frequency sweep. A reference measurement was thus taken at each step of the sweep and used to deduce the absolute value of the transmission.

Figure $6 \mathrm{~b}$ shows the transmission of water vapour in air (partial pressure of water vapour in air $P_{\mathrm{H}_{2} \mathrm{O}}=1.63 \mathrm{kPa}$, total pressure $P_{\text {tot }}=101.3 \mathrm{kPa}, T=20^{\circ} \mathrm{C}$ ) measured with the dualcomb set-up as well as a transmission simulation using HITRAN database $^{43}$ (see Methods). Figure $6 c$ shows the frequency sweep applied to the sample comb, measured on the multi-heterodyne beat signal. The wavenumber scale calibration was carried out by using the HITRAN simulation and by applying a rigid shift to the measured transmission spectrum in order to fit the HITRAN simulation spectrum (see Supplementary Methods). Over the entire duration of the measurement (few hours), the slow drifts reduce the quality of the interleaved transmission spectrum and a moving average filter was used for smoothing the interleaved transmission spectrum, reducing the effective resolution to $800 \mathrm{MHz}$. A typical interleaved spectrum contains $\sim 5,000$ points and the length of the filter was set to 11 points (see Supplementary Methods).

A clear agreement between the two measurements is observed over the entire measurement bandwidth $\left(16 \mathrm{~cm}^{-1}\right)$ and the absolute value of the transmission could also be retrieved. Owing to the important attenuation of the comb lines situated in the 
a

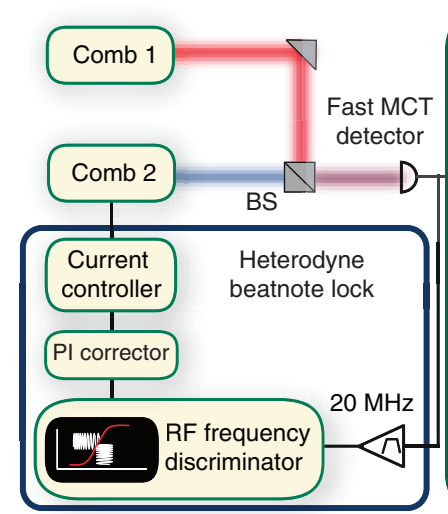

C

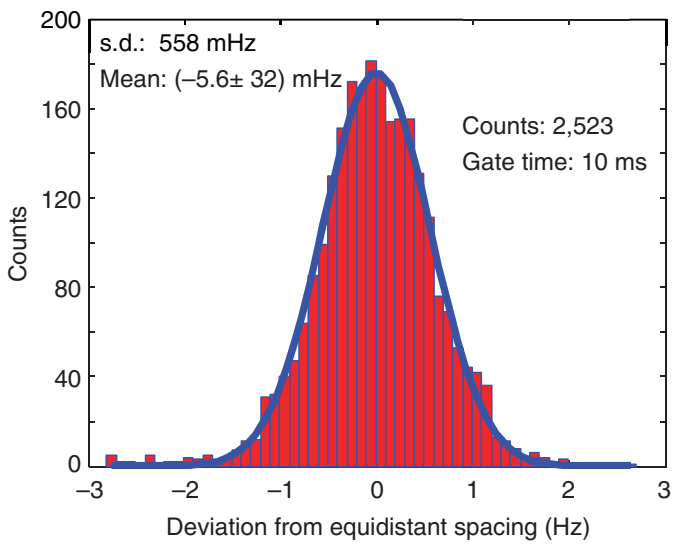

b
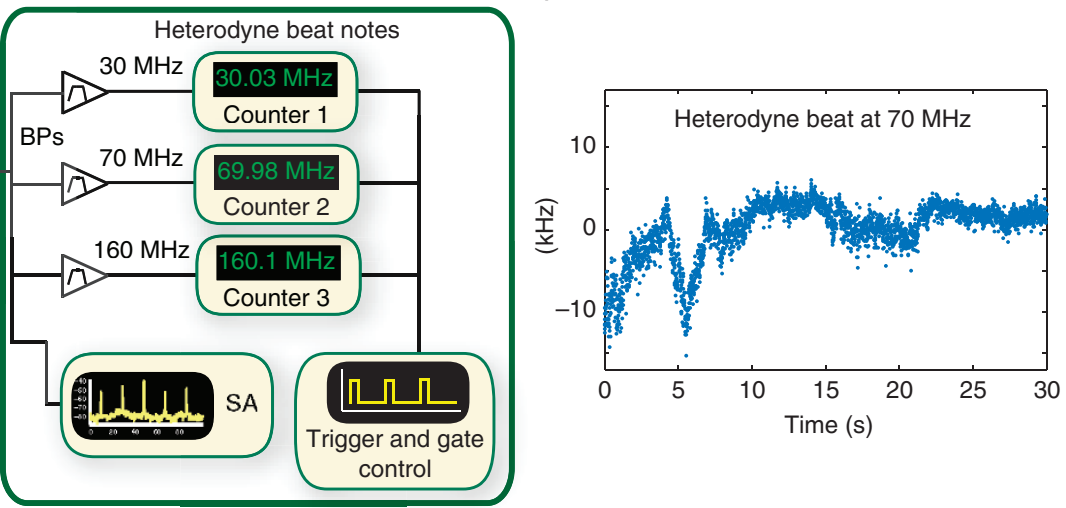

d

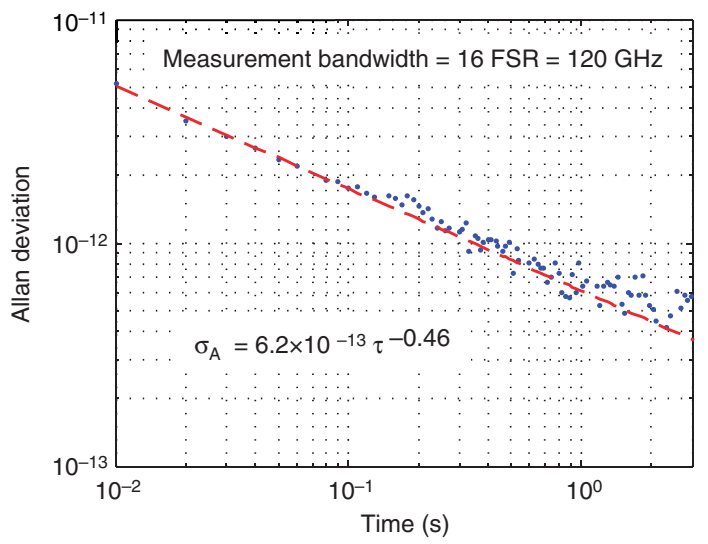

Figure 4 | Measurement of the deviation from equidistant mode spacing. (a) Schematic representation of the set-up used for measuring the deviation from equidistant mode spacing $\epsilon$ based on a multi-heterodyne measurement. SA, spectrum analyser; BPs, band-pass filters; BS, beam splitter; $\mathrm{PI}$, proportional integral; $\mathrm{MCT}$, mercury cadmium telluride. (b) Frequency oscillations of a single multi-heterodyne beat note when the slow drifts are corrected by the stabilization loop, measured with a frequency counter (total measurement time $=30 \mathrm{~s}$, gate time $=10 \mathrm{~ms}$ ). (c) Distribution of the deviation from equidistance mode spacing $\epsilon$. Total measurement time $=25.23 \mathrm{~s}$. Gate time $=10 \mathrm{~ms}$. The distribution shows a gaussian distribution with an average value of $(-5.6 \pm 32) \mathrm{mHz}$ and a s.d. of $558 \mathrm{mHz}$. (d) Allan deviation of $\epsilon$ normalized by the total bandwidth of the measurement $(16 \times \mathrm{FSR}=120 \mathrm{GHz})$. The Allan deviation shows an inverse square root dependency.

a

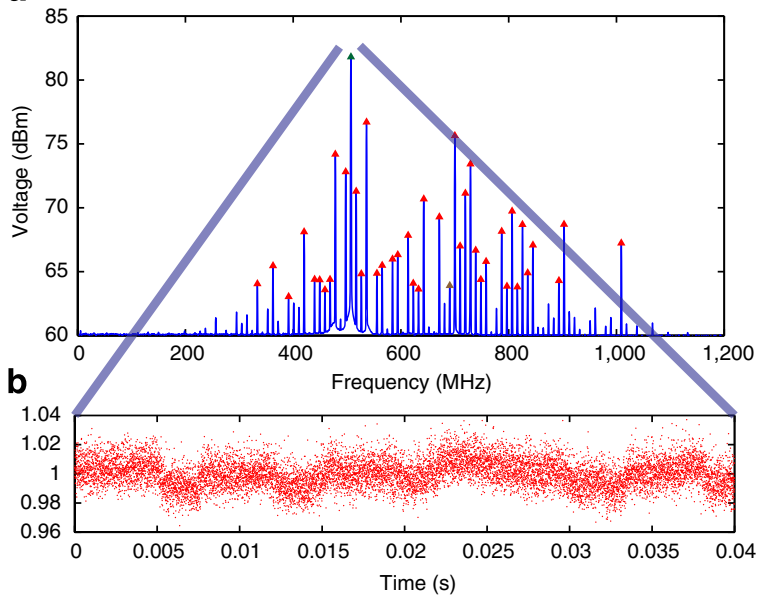

C

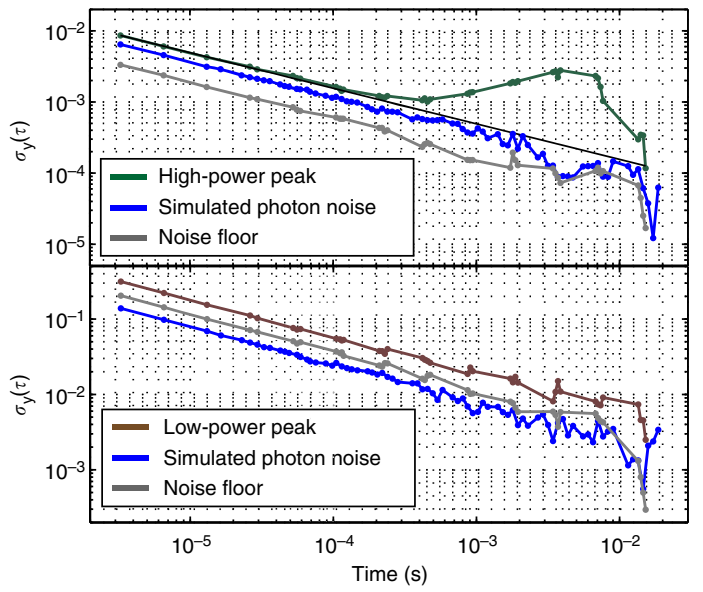

Figure 5 | Amplitude noise of the multi-heterodyne beat notes. (a) Multi-heterodyne spectra used for the amplitude noise measurement. The peak numbers 11 and 24 are highlighted as they were used for the amplitude noise characterization. (b) Amplitude oscillations of the amplitude of the highest amplitude beat note, normalized by its mean value (11th peak, in green). The time resolution of the measurement is again set to $3.2 \mu \mathrm{s}$. (c) Top: normalized Allan variation of the amplitude variations of the highest amplitude beat notes (11th peak, in green) as a function of the integration time. Bottom: normalized Allan variation of the amplitude variations of the low-amplitude beat notes (24th peak, in brown) as a function of the integration time. In both graphs we represent the Allan deviation of the amplitude variations induced by the detection noise floor and by the simulated photon noise. 
a

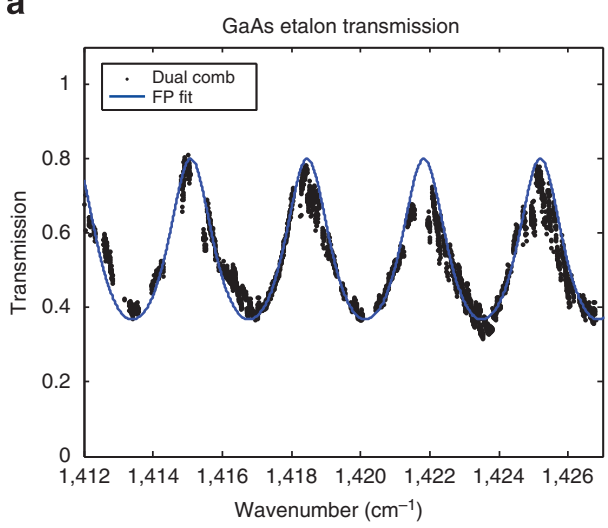

C

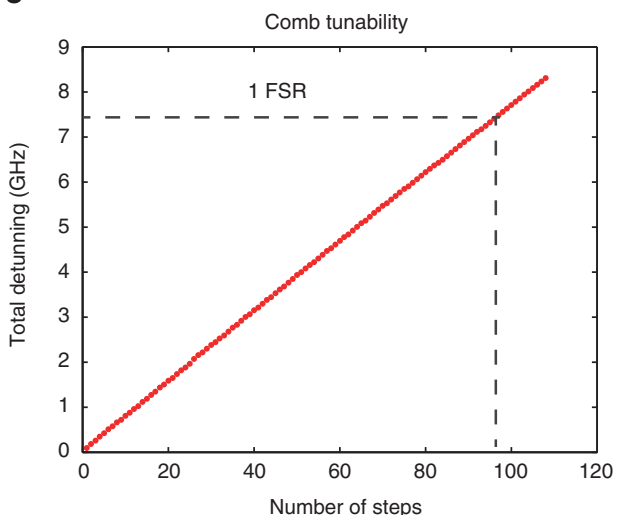

b

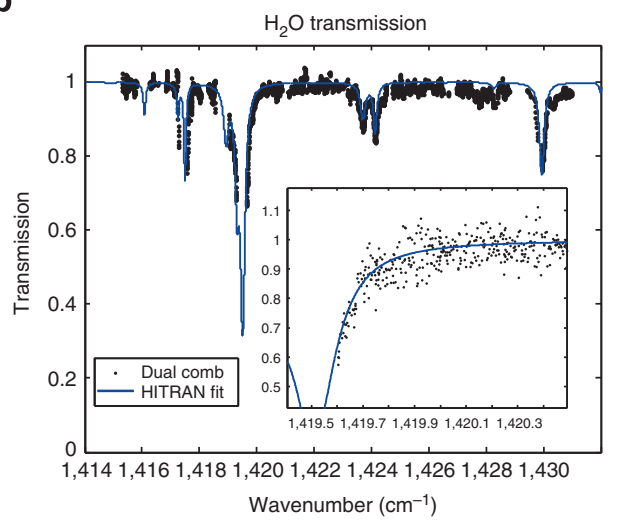

d

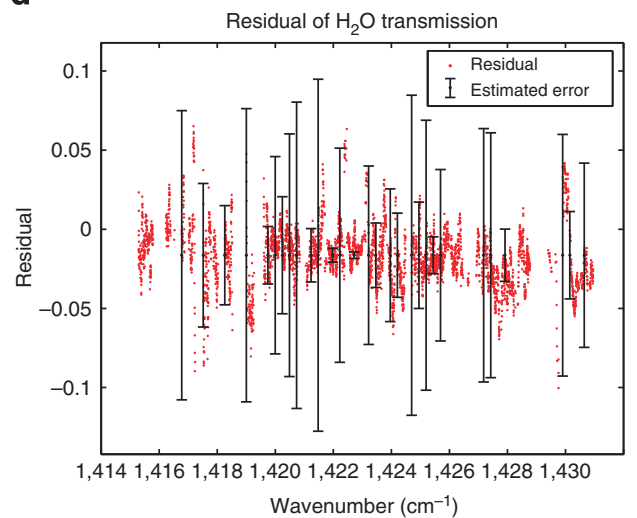

Figure 6 | Dual-comb transmission spectra. (a) GaAs Etalon transmission spectrum measured with our dual-comb spectrometer (0.25-cm ${ }^{-1} \mathrm{comb}^{-}$ spacing swept with a 80-MHz step) together with a fit based on the expression of the transmission of an etalon. (b) Water vapour transmission spectra in air $\left(P_{\mathrm{H}_{2} \mathrm{O}}=1.63 \mathrm{kPa}\right.$, total pressure $\left.P_{\text {tot }}=101.3 \mathrm{kPa}, T=20^{\circ} \mathrm{C}\right)$ measured with our dual-comb spectrometer $(800 \mathrm{MHz}$ of spectral resolution after averaging) and HITRAN simulation. Inset: expansion of the measured transmission over $\sim 1 \mathrm{~cm}^{-1}$ with the full resolution ( $80 \mathrm{MHz}$ ). (c) $\mathrm{Values}$ of frequency tuning of the sample comb for each step of the tuning. The total detuning exceeded the bandwidth corresponding to the value of one comb line spacing $(\sim 7.5 \mathrm{GHz})$. (d) Residual between the water vapour transmission and the simulation using HITRAN database as well as the estimated error $\sigma_{T}$.

vicinity of the water absorption lines, their attenuated amplitude could lie below the noise floor of the detection set-up. In such a case, the algorithm calculating the absorption will not be able to retrieve the entire shape of the absorption line. This can be observed on the shape of the water absorption line situated at $1,419.5 \mathrm{~cm}^{-1}$. Finally, as the multi-heterodyne spectrum presents beat notes with difference in amplitudes of more than $40 \mathrm{~dB}$, the very low-intensity beat notes will not be detected by the algorithm calculating the absorption. This results in some holes on the spectrum and can also be observed on Fig. 6b, for instance, close to the water absorption line situated at $1,416.3 \mathrm{~cm}^{-1}$. This issue could be resolved by sweeping the combs by more than one comb spacing.

The residual between the water vapour measured with the dual-comb set-up and the HITRAN database simulation was also computed. Figure $6 \mathrm{~d}$ shows the residual of all the points of the interleaved transmission spectrum together with the error estimated from the amplitude noise considerations (see Methods). The error calculation correctly predicts the residual between our measurement results and the HITRAN simulation, although the measurement is still clearly limited by technical noise. More importantly, the distribution of the residuals has a s.d. of 0.014 , showing that our measurement has a precision of $1.4 \%$. Compared with the transmission measurements demonstrated in ref. 41, we have demonstrated that the comb operation of QCLs is crucial for achieving broadband multi-heterodyne spectroscopy.

\section{Discussion}

In conclusion, we have demonstrated a compact, semiconductorbased mid-infrared dual-comb spectrometer based on QCL frequency combs. We deeply characterized the noise properties of the dual-comb spectrometer. The experiment was also used to demonstrate the equidistance of the comb lines down to a fractional accuracy of $7.5 \times 10^{-16}$. As a proof of principle, we have demonstrated broadband $\left(16 \mathrm{~cm}^{-1}\right)$ high-resolution $\left(80 \mathrm{MHz}=0.0027 \mathrm{~cm}^{-1}\right)$ absorption spectroscopy of a lowfinesse etalon as well as water vapour in air. These proof of principle experiments will encourage further development of instruments with much higher performances. Better-matched QCL combs implementing dispersion compensation ${ }^{44}$ should enable a great increase in spectral coverage up to hundreds of $\mathrm{cm}^{-1}$ and decrease the peak-to-peak intensity variation. Better optics and detectors with a larger dynamical range will better deal with the line-to-line amplitude variation. Actively stabilization of both the frequency comb offset frequency and repetition frequency will also improve the SNR of our measurements as well as simplifying the data treatment. All-electrical measurements and stabilization of the repetition frequency of the laser could be carried out as the intermode beat note can be measured electrically. Our system holds the potential to achieve a monolithically integrated mid-infrared dual-comb spectrometer achieving ultrahigh sensitivity combined with broadband spectral coverage and high resolution, with applications in many fields such as personalized medicine, environment and process control. 


\section{Methods}

Dual-comb set-up. The QCLs used to realize the dual-comb spectroscopy set-up were not cleaved together and therefore present different comb spacings. The difference between two comb spacings being $\Delta f=\frac{c \Delta L}{2 n_{\mathrm{g}} L^{2}}=f \frac{\Delta L}{L}$, where $n_{\mathrm{g}}$ is the group index, a difference of the two comb spacings $\Delta f \leq 50 \mathrm{MHz}$ corresponds, for a $L=6$-mm-long device, to a maximum length difference $\Delta L$ between the two devices of less than $40 \mu \mathrm{m}$. The frequency difference $\Delta f$ could be set between 5 and $40 \mathrm{MHz}$ by controlling the current and/or temperature of each comb.

Each comb is first collimated by an 1-inch $\mathrm{f} / 1$ aspheric lens. The sample to be investigated by our dual-comb spectroscopy set-up is injected in a gas cell (6-cm long with AR-coated 2-inch ZnSe windows). The gas cell is also placed in a motorized stage and can be moved out of the optical path. Otherwise, a low-finesse GaAs etalon is placed on the motorized stage. Both combs are heterodyned into a fast $(590 \mathrm{MHz}$, $3 \mathrm{~dB}$ cutoff frequency) thermoelectric cooled (TEC) mercury cadmium telluride (MCT) photovoltaic detector (Vigo PV-4TE-10.6) giving the sample signal. For reference measurements (without sample), the sample and LO combs are both separated by $50-50$ wedged beam splitters and heterodyned in a second identical detector without the influence of the investigated sample. The beams were attenuated by neutral density filters to avoid saturation of the detectors. Both signals are then acquired either with a spectrum analyser or by a 12-bit vertical resolution, $1 \mathrm{GHz}$ analogue bandwidth, 2.5 GSample/s sampling frequency oscilloscope (memory of 250 MSamples for the XL memory option (used for Fig. 3a) or 100 MSamples per channel for L memory option (used for all other figures)). Both sample signal and reference signal can be acquired with the same trigger on the two different channels of the oscilloscope. The entire set-up is placed in a nitrogen-purged environment. All technical sources of noise on the set-up were carefully investigated and reduced. Acoustic noise as well as temperature fluctuations of the room are reduced by placing the entire set-up into a box. Both lasers are driven with low noise current drivers (Wavelength electronics QCL1000) with a specified average current noise density of $2 \mathrm{nA} / \sqrt{\mathrm{Hz}}$. In addition, a home-made battery system capable of driving both lasers for several hours was used. The temperature fluctuations of the lasers were also reduced to less than $10 \mathrm{mK}$ by using a low thermal drift temperature controller (wavelength electronics PTC10K-CH) with a $50-\mathrm{k} \Omega$ thermistor.

Measurement of the deviation from equidistance mode spacing. The multiheterodyne signal is detected by a fast MCT photovoltaic detector (Vigo PV-4TE10.6). Three narrow bandwidth $(5 \mathrm{MHz})$ band-pass filters centred at 30,70 and $160 \mathrm{MHz}$ are used to isolate the beat notes. Three frequency counters (Agilent $53220 \mathrm{~A}$ ) are used to count for the frequency oscillations of the beat notes. An active stabilization was implemented to perform a weak locking $(\sim 1 \mathrm{kHz}$ bandwidth) for correcting the multi-heterodyne beat spectrum against the slow drifts. A band-pass filter (20 MHz centre frequency, $5 \mathrm{MHz}$ bandwidth) was used to isolate a fourth beat note to be used as error signal. The frequency oscillations of this beat note were converted into amplitude oscillations through a RF frequency discriminator (Miteq FMDM-21.4/4-2B-R, centre frequency $21.4 \mathrm{MHz}, 8 \mathrm{MHz}$ bandwidth, $1 \mathrm{~V} \mathrm{MHz}^{-1}$ sensitivity). The output of this RF frequency discriminator was used as the input of a Proportional Integral corrector (Zurich Instrument HH2LI-PID, $5 \mathrm{kHz}$ bandwidth) acting on the current of one of the combs. High SNR ( $30 \mathrm{~dB}$ or more) beat notes were used for this experiment in order to be correctly detected by the frequency counters. The gate and the time control were controlled by a trigger (Agilent $33220 \mathrm{~A}$, pulse mode). To verify that the counters were correctly synchronized, a FM signal with a slowly varying carrier frequency (carrier frequency $10 \mathrm{MHz}$, frequency deviation $\sim 1 \mathrm{kHz}$, FM frequency $\sim 1 \mathrm{~Hz}$ ) was generated by a frequency generator (Agilent 33220 A, FM modulation mode) and counted by the three counters. The deviation from the equidistant spacing was measured and showed values at least one order of magnitude lower than the deviation from the equidistant spacing measured with the QCL combs. All the instruments where connected to the same $10-\mathrm{MHz}$ clock reference.

Allan deviation measurements. The Allan deviation of the noise floor is evaluated by blocking the two beams, measuring the amplitude oscillations at the frequency of the corresponding beat note and normalizing these oscillations by the mean amplitude of the corresponding beat note. The Allan deviation of the photon noise accounts for the amplitude oscillations of the beat note in the case where the only noise source is the shot noise of the lasers (see Supplementary Note 2).

HITRAN simulation. The HITRAN 2008 database was used for the simulation of the transmission of water vapour in air. The partial pressure of water vapour in air used in the simulations is $P_{\mathrm{H}_{2} \mathrm{O}}=1.63 \mathrm{kPa}$, the total pressure is $P_{\mathrm{tot}}=101.3 \mathrm{kPa}$ and the temperature is $T=20^{\circ} \mathrm{C}$. The frequency resolution was set to $0.001 \mathrm{~cm}^{-1}$. A Voigt profile was assumed and no instrumental lineshape was taken into account.

Residual and error estimation. The transmission of water vapour is related to the voltage generated by a heterodyne beat on the detector by:

$$
T=\left(\frac{V_{\mathrm{H}_{2} \mathrm{O}}}{V_{\mathrm{N}_{2}}}\right)^{2}
$$

where $T$ is the transmission, $V_{\mathrm{H}_{2} \mathrm{O}}$ (respectively $V_{\mathrm{N}_{2}}$ ) is the voltage generated by a multi-heterodyne beat with the gas cell filled with water vapour $\left(N_{2}\right)$. Therefore, any voltage oscillation of $V_{\mathrm{H}_{2} \mathrm{O}}\left(V_{\mathrm{N}_{2}}\right)$, characterized by its s.d. $\sigma_{V_{\mathrm{H}_{2} \mathrm{O}}}\left(\sigma_{V_{\mathrm{N}_{2}}}\right)$ influences the transmission $t$ by:

$$
\frac{\sigma_{T}}{T}=\sqrt{\left(2 \frac{\sigma_{V_{\mathrm{H}_{2} \mathrm{O}}}}{V_{\mathrm{H}_{2} \mathrm{O}}}\right)^{2}+\left(2 \frac{\sigma_{V_{\mathrm{N}_{2}}}}{V_{\mathrm{N}_{2}}}\right)^{2}}
$$

where $\sigma_{t}$ is the s.d. of the transmission.

We assume that $\frac{\sigma_{V_{\mathrm{H}_{2}} \mathrm{O}}}{V_{\mathrm{H}_{2} \mathrm{O}}}=\frac{\sigma_{V_{\mathrm{N}_{2}}}}{V_{\mathrm{N}_{2}}}$ and we estimate $\frac{\sigma_{V_{\mathrm{N}_{2}}}}{V_{\mathrm{N}_{2}}}$ by measuring the s.d. $\sigma_{V_{\mathrm{N}_{2}}}$ and the mean value $V_{\mathrm{N}_{2}}$ of the voltage oscillations created by every heterodyne beat note, as shown in Fig. $5 \mathrm{~b}$.

\section{References}

1. Udem, T., Holzwarth, R. \& Hänsch, T. W. Optical frequency metrology. Nature 416, 233-237 (2002).

2. Diddams, S. A. The evolving optical frequency comb. JOSA B 27, B51-B62 (2010).

3. Schiller, S. Spectrometry with frequency combs. Opt. Lett. 27, 766-768 (2002).

4. Keilmann, F., Gohle, C. \& Holzwarth, R. Time-domain mid-infrared frequency-comb spectrometer. Opt. Lett. 29, 1542-1544 (2004).

5. Coddington, I., Swann, W. \& Newbury, N. Coherent multiheterodyne spectroscopy using stabilized optical frequency combs. Phys. Rev. Lett. 100, 013902 (2008).

6. Bernhardt, B. et al. Cavity-enhanced dual-comb spectroscopy. Nat. Photon 4, 55-57 (2010)

7. Coddington, I., Swann, W. \& Newbury, N. Coherent dual-comb spectroscopy at high signal-to-noise ratio. Phys. Rev. A. 82, 043817 (2010).

8. Roy, J., Deschênes, J.-D., Potvin, S. \& Genest, J. Continuous real-time correction and averaging for frequency comb interferometry. Opt. Express 20, 21932-21939 (2012).

9. Zolot, A. et al. Direct-comb molecular spectroscopy with accurate, resolved comb teeth over 43 thz. Opt. Lett. 37, 638-640 (2012).

10. Griffiths, P. \& De Haseth, J. A. Fourier Transform Infrared Spectrometry vol. 171John Wiley \& Sons, 2007).

11. Schliesser, A., Picqué, N. \& Hänsch, T. W. Mid-infrared frequency combs. Nat. Photon 6, 440-449 (2012).

12. Sorokin, E., Tolstik, N. \& Sorokina, I. Kerr-lens mode-locked cr: Zns laser. Opt. Lett. 38, 299-301 (2013).

13. Sorokin, E., Tolstik, N., Schaffers, K. I. \& Sorokina, I. T. Femtosecond sesammodelocked cr: Zns laser. Opt. Express 20, 28947-28952 (2012).

14. Solodyankin, M. A. et al. Mode-locked $1.93 \mu \mathrm{m}$ thulium fiber laser with a carbon nanotube absorber. Opt. Lett. 33, 1336-1338 (2008).

15. Fischer, C. \& Sigrist, M. W. Solid-state Mid-Infrared Laser Sources 99-143 (Springer, 2003).

16. Schliesser, A., Brehm, M., Keilmann, F. \& van der Weide, D. Frequency-comb infrared spectrometer for rapid, remote chemical sensing. Opt. Express 13, 9029-9038 (2005).

17. Maddaloni, P., Malara, P., Gagliardi, G. \& De Natale, P. Mid-infrared fibrebased optical comb. New J. Phys. 8, 262 (2006).

18. Baumann, E. et al. Spectroscopy of the methane $v_{3}$ band with an accurate midinfrared coherent dual-comb spectrometer. Phys. Rev. A. 84, 062513 (2011)

19. Vodopyanov, K. L., Sorokin, E., Sorokina, I. T. \& Schunemann, P. G. Mid-IR frequency comb source spanning 4.4-5.4 $\mu \mathrm{m}$ based on subharmonic GaAs optical parametric oscillator. Opt. Lett. 36, 2275-2277 (2011).

20. Galli, I. et al. High-coherence mid-infrared frequency comb. Opt. Express. 21, 28877-28885 (2013).

21. Del'Haye, P. et al. Optical frequency comb generation from a monolithic microresonator. Nature 450, 1214-1217 (2007).

22. Wang, C. et al. Mid-infrared optical frequency combs at $2.5 \mu \mathrm{m}$ based on crystalline microresonators. Nat. Commun. 4, 1345 (2013).

23. Griffith, A. et al. Silicon-Chip Mid-Infrared Frequency Comb Generation. Preprint at http://arxiv.org/abs/1408.1039 (2014).

24. Ideguchi, T., Bernhardt, B., Guelachvili, G., Hänsch, T. W. \& Picqué, N. Raman-induced kerr-effect dual-comb spectroscopy. Opt. Lett. 37, 4498-4500 (2012).

25. Ideguchi, T. et al. Coherent raman spectro-imaging with laser frequency combs. Nature 502, 355-358 (2013).

26. Faist, J. et al. Quantum cascade laser. Science 264, 553-556 (1994).

27. Beck, M. et al. Continuous wave operation of a mid-infrared semiconductor laser at room temperature. Science 295, 301-305 (2002).

28. Lyakh, A., Maulini, R., Tsekoun, A., Go, R. \& Patel, C. K. N. Tapered $4.7 \mu \mathrm{m}$ quantum cascade lasers with highly strained active region composition delivering over 4.5 watts of continuous wave optical power. Opt. Express 20, 4382-4388 (2012).

29. Gmachl, C., Sivco, D. L., Colombelli, R., Capasso, F. \& Cho, A. Y. Ultra-broadband semiconductor laser. Nature 415, 883-887 (2002). 
30. Hugi, A., Maulini, R. \& Faist, J. External cavity quantum cascade laser. Semicond. Sci. Technol. 25, 083001 (2010).

31. Faist, J., Beck, M., Aellen, T. \& Gini, E. Quantum-cascade lasers based on a bound-to-continuum transition. Appl. Phys. Lett. 78, 147 (2001).

32. Wang, C. Y. et al. Mode-locked pulses from mid-infrared quantum cascade lasers. Opt. Express 17, 12929-12943 (2009).

33. Gordon, A. et al. Multimode regimes in quantum cascade lasers: From coherent instabilities to spatial hole burning. Phys. Rev. A. 77, 053804 (2008).

34. Wójcik, A. K. et al. Generation of picosecond pulses and frequency combs in actively mode locked external ring cavity quantum cascade lasers. Appl. Phys. Lett. 103, 231102 (2013).

35. Barbieri, S. et al. Coherent sampling of active mode-locked terahertz quantum cascade lasers and frequency synthesis. Nat. Photon 5, 306-313 (2011)

36. Hugi, A., Villares, G., Blaser, S., Liu, H. \& Faist, J. Mid-infrared frequency comb based on a quantum cascade laser. Nature 492, 229-233 (2012).

37. Friedli, P. et al. Four-wave mixing in a quantum cascade laser amplifier. Appl. Phys. Lett. 102, 222104 (2013).

38. Khurgin, J. B., Dikmelik, Y., Hugi, A. \& Faist, J. Coherent frequency combs produced by self frequency modulation in quantum cascade lasers. Appl. Phys. Lett. 104, 081118 (2014).

39. Paiella, R. et al. Self-mode-locking of quantum cascade lasers with giant ultrafast optical nonlinearities. Science 290, 1739-1742 (2000).

40. Newbury, N., Coddington, I. \& Swann, W. Sensitivity of coherent dual-comb spectroscopy. Opt. Express 18, 7929-7945 (2010).

41. Wang, Y., Soskind, M. G., Wang, W. \& Wysocki, G. High-resolution multiheterodyne spectroscopy based on fabry-perot quantum cascade lasers. Appl. Phys. Lett. 104, 031114 (2014)

42. Henry, C., Henry, P. \& Lax, M. Partition fluctuations in nearly singlelongitudinal-mode lasers. J. Lightw. Technol. 2, 206-216 (1984).

43. Rothman, L. S. et al. The HITRAN 2008 molecular spectroscopic database. J. Quant. Spectrosc. Radiati. Transfer 110, 533-572 (2009).
44. Burghoff, D. et al. Terahertz laser frequency combs. Nat. Photon 8, 462-467 (2014).

\section{Acknowledgements}

We thank F. Cappelli for his help with the equidistance mode spacing measurements, B Tuzson and S. Schilt for useful discussions. We acknowledge Y. Bonetti for his help with the manuscript illustration. This work was financially supported by the Swiss National Science Foundation. We also thank the ETH Pioneer Fellowship programme for financial support of this project.

\section{Author contributions}

G.V. constructed the experimental set-up. G.V. and A.H. carried out the measurements, analysed the data and developed the algorithm for interleaving the dual-comb transmission spectra. S.B. provided both quantum-cascade-laser frequency combs. G.V. wrote the paper and made the figures. G.V., A.H. and J.F. joined the discussion and provided comments. All the work has been carried out under the supervision of J.F.

\section{Additional information}

Supplementary Information accompanies this paper at http://www.nature.com/ naturecommunications

Competing financial interests: The authors declare no competing financial interests.

Reprints and permission information is available online at http://npg.nature.com/ reprintsandpermissions/

How to cite this article: Villares, G. et al. Dual-comb spectroscopy based on quantumcascade-laser frequency combs. Nat. Commun. 5:5192 doi: 10.1038/ncomms6192 (2014). 